\title{
Large Deviations for Random Walks in a Mixing Random Environment and Other (Non-Markov) Random Walks
}

\author{
FIRAS RASSOUL-AGHA \\ The Ohio State University
}

\begin{abstract}
We extend a recent work by S. R. S. Varadhan [8] on large deviations for random walks in a product random environment to include more general random walks on the lattice. In particular, some reinforced random walks and several classes of random walks in Gibbs fields are included. (c) 2004 Wiley Periodicals, Inc.
\end{abstract}

\section{Introduction}

A process $P_{x}$, taking values in $\left(\mathbb{Z}^{d}\right)^{\mathbb{N}}$, is called a random walk on $\mathbb{Z}^{d}$ starting at $x$, if there exists a function $q$ on $\bigcup_{n \geq 0}\left(\mathbb{Z}^{d}\right)^{n} \times \mathbb{Z}^{d}$ such that

$$
\begin{aligned}
P_{x}\left(X_{0}=x\right) & =1, \\
P_{x}\left(X_{n+1}=X_{n}+z \mid \mathcal{A}_{n}\right) & =q\left(\left(z_{1}, \ldots, z_{n}\right), z\right),
\end{aligned}
$$

where $\mathcal{A}_{n}=\left\{X_{1}-X_{0}=z_{1}, \ldots, X_{n}-X_{n-1}=z_{n}\right\}$. Throughout this paper, we will use $z_{j}$ 's and $x_{j}$ 's interchangeably, where $z_{j}=x_{j}-x_{j-1}$ and $x_{0}=x$ (the starting point).

In part of this work, namely Sections 4 and 5, we focus on a special kind of random walk, which is a random walk in a mixing random environment (RWRE). In this model, an environment is a collection of transition probabilities

$$
\omega=\left(\pi_{x y}\right)_{x, y \in \mathbb{Z}^{d}} \in[0,1]^{\mathbb{Z}^{d} \times \mathbb{Z}^{d}} \quad \text { with } \sum_{y \in \mathbb{Z}^{d}} \pi_{x y}=1 .
$$

Let us denote by $\Omega$ the space of all such transition probabilities. The space $\Omega$ is equipped with the canonical product $\sigma$-field $\mathfrak{S}$ and with the natural shift $\left(\theta_{z} \omega\right)_{x, y}=$ $\omega_{x+z, y+z}$ for $z \in \mathbb{Z}^{d}$. Here, $\omega_{x y}$ stands for the $(x, y)^{\text {th }}$ coordinate of $\omega \in \Omega$. We will also use $\omega_{x}=\left(\omega_{x y}\right)_{y \in \mathbb{Z}^{d}}$. On the space of environments $(\Omega, \mathfrak{S})$, we are given a certain $\theta$-invariant probability measure $\mathbb{P}$ with $\left(\Omega, \mathfrak{S},\left(\theta_{z}\right)_{z \in \mathbb{Z}^{d}}, \mathbb{P}\right)$ ergodic. We will say that the environment is i.i.d. when $\mathbb{P}$ is a product measure.

Let us now describe the process. First, the environment $\omega$ is chosen from the distribution $\mathbb{P}$. Once this is done, it remains fixed for all times. The random walk 
in environment $\omega$ is then the canonical Markov chain $\left(X_{n}\right)_{n \geq 0}$ with state space $\mathbb{Z}^{d}$ and transition probability

$$
\begin{aligned}
P_{0}^{\omega}\left(X_{0}=0\right) & =1, \\
P_{0}^{\omega}\left(X_{n+1}=y \mid X_{n}=x\right) & =\pi_{x y}(\omega) .
\end{aligned}
$$

The process $P_{0}^{\omega}$ is called the quenched law. The annealed law is then

$$
P_{0}=\int P_{0}^{\omega} d \mathbb{P}(\omega) .
$$

The marginal of $P_{0}$ on the space of walks is in fact a random walk on $\mathbb{Z}^{d}$.

To see this, we need to introduce the number of visits of the walk $\mathrm{w}=$ $\left(z_{1}, \ldots, z_{n}\right)$, starting at 0 , to site $x$ in direction $z$, as

$$
n_{x z}(\mathrm{w})=\sum_{j=0}^{n-1} \mathbb{I}_{\left\{x_{j}=x, x_{j+1}=x+z\right\}}(\mathrm{w}) \text {. }
$$

Indeed, one has

$$
q(\mathrm{w}, z)=\mathbb{E}^{\mathbb{P}}\left(\frac{\prod_{x, y} \pi_{x, x+y}^{n_{x y}(\mathrm{w})}(\omega)}{\mathbb{E}^{\mathbb{P}}\left(\prod_{x, y} \pi_{x, x+y}^{n_{x y}(\mathrm{w})}\right)} \pi_{x_{n}, x_{n}+z}(\omega)\right) .
$$

In [8] Varadhan shows the existence of a convex, lower-semicontinuous rate function for large deviations of the position $X_{n}$ under the annealed measure $P_{0}$, when $\mathbb{P}$ is i.i.d. and satisfies the regularity condition.

Hypothesis A There exists a deterministic function $p_{0}: \mathbb{Z}^{d} \rightarrow[0,1]$ and three deterministic constants $-M>0$ (the range of the increments), $\kappa \in(0,1)$ (the ellipticity constant), and $c>0$ - such that $p_{0}(z)=0$ for $|z|>M, p_{0}(e)>\kappa$ for $|e|=1$, and for all $z \in \mathbb{Z}^{d}$

$$
\mathbb{P}\left(p_{0}(z) \leq \pi_{0, z} \leq c p_{0}(z)\right)=1 .
$$

Here and in the rest of the paper, $|\cdot|$ will denote the $\ell^{1}$ distance on $\mathbb{Z}^{d}$ so that $M=1$ means the walk is nearest-neighbor. The analogous regularity condition for general random walks would be the following:

Hypothesis $\mathrm{A}^{\prime}$ There exists a deterministic function $p_{0}: \mathbb{Z}^{d} \rightarrow[0,1]$ and three deterministic constants $M>0, \kappa \in(0,1)$, and $c>0$ such that $p_{0}(z)=0$ for $|z|>$ $M, p_{0}(e)>\kappa$ for $|e|=1$, and for all $z \in \mathbb{Z}^{d}$ and $\mathrm{w}=\left(z_{1}, \ldots, z_{n}\right) \in \bigcup_{m \geq 0}\left(\mathbb{Z}^{d}\right)^{m}$ with $\left|z_{j}\right| \leq M$, when $1 \leq j \leq n$,

$$
p_{0}(z) \leq q(\mathrm{w}, z) \leq c p_{0}(z) .
$$

In the present paper we are interested in extending the result in [8] to the noni.i.d. case. The regularity condition will still be assumed to hold. We will use the approach of the point of view of the particle, where we look at everything from the walker's point of view. The advantage of this approach is that it overcomes the non-Markovian character of the annealed process. However, one then has to 
deal with the larger state space and the long memories of the walker. In [8] the author overcomes these difficulties by using estimates, some of which depend on the hypothesis that $\mathbb{P}$ is a product measure. We will show that these estimates in fact hold for more general environments. In fact, we will show that the method, introduced in [8], is general enough to include many other random walks.

In Section 2 we introduce further notation and describe the approach of the point of view of the particle, mentioned above. We also state Theorem 2.1, which just adapts the result of [8] to the case of general random walks. We then roughly sketch the ideas of the proof, which is basically the same as in [8], and point out the problems that need to be tackled in order to generalize the result of [8]. For example, while $q$ is well-defined for paths of finite length, defining transition probabilities for the process of paths, shifted to be seen from the origin, is no longer obvious, if the walk has a long history.

In Section 3 we give two examples of random walks other than random walks in a random environment. Namely, we look at some reinforced random walks on $\mathbb{Z}^{d}$. We show that a large-deviations principle for the position is satisfied, and we discuss the zero set of the rate function.

In Section 4 we shift our interest to random walks in a mixing random environment. We introduce our mixing assumptions and define the transition probabilities, mentioned above.

In Section 5 we state and prove our main theorem (Theorem 5.1), which is an application of Theorem 2.1 to the case of random walks in certain Gibbs fields.

\section{The Large-Deviations Principle}

The method of the point of view of the particle consists of describing everything, that is, the path and the environment (for RWRE), as seen from the walker. Consider a walk of $n \geq 0$ steps $\left\{x_{0}=0, x_{1}, \ldots, x_{n}\right\}$. Then, in the case of RWRE, the environment, as seen from the walker at $x_{n}$, would be $\omega_{n}=\theta_{x_{n}} \omega$. Also, in the more general setup of general random walks, the path, as seen from $x_{n}$, would be

$$
\left\{x_{-n}=-x_{n}, x_{-n+1}=x_{1}-x_{n}, \ldots, x_{0}=0\right\} .
$$

Since we know the path ends at 0 , we will instead consider the increments

$$
\mathrm{w}_{n}=\left(z_{-n+1}=x_{-n+1}-x_{-n}, \ldots, z_{0}=x_{0}-x_{-1}\right) .
$$

The collection of such paths will be denoted by $\mathrm{W}_{n}=\{e:|e| \leq M\}^{n}$. Note that $\mathrm{W}_{0}$ has only one element, which we will denote by $\phi$. Furthermore, define the space $\mathrm{W}_{\infty}=\{e:|e| \leq M\}^{-\mathbb{N}}$ of walks of infinite length ending at 0 . Here $-\mathbb{N}=\{0,-1,-2,-3, \ldots\}$. Let $\mathrm{W}=\bigcup_{n \geq 0} \mathrm{~W}_{n} \cup \mathrm{W}_{\infty}$, and define $x_{j}(\mathrm{w})$ as in (2.1) when $-n \leq j \leq 0$. Also, if $n<\infty$, define $x_{j}(\mathrm{w})=\Delta$ (the cemetery state) for $j<-n$ and $\mathrm{w} \in \mathrm{W}_{n}$. Similarly, for $n<\infty, z_{j}=S$ (for "stop") when $j \leq-n$ and $\mathrm{w} \in \mathrm{W}_{n}$. 
Next, we define the number of visits to site $x$, in direction $z$, as

$$
n_{x z}(\mathrm{w})=\sum_{j \leq-1} \mathbb{I}_{\left\{x_{j}=x, x_{j+1}=x+z\right\}}(\mathrm{w}),
$$

and then $n_{x}=\sum_{z} n_{x z}$ is the number of visits to $x$, which might be finite or infinite. Now, let us turn to the dynamics on these spaces. This will lead us to the first problem, caused by the long memory of the process, for example, when considering random walks in mixing random environments.

First, for $|z| \leq M$ and $\mathrm{w} \in \mathrm{W}$, define the shifts $T^{*}$ and $T_{z}$ so that $z_{j+1}\left(T^{*}(\mathrm{w})\right)=$ $z_{j}(\mathrm{w}), z_{0}\left(T_{z}(\mathrm{w})\right)=z$, and $z_{j}\left(T_{z}(\mathrm{w})\right)=z_{j+1}(\mathrm{w})$ for $j \leq-1$. Then any random walk, defined by a given $q$, induces a Markov process $Q_{\mathrm{w}}$ on the space $\bigcup_{n} \mathrm{~W}_{n}$. Indeed, starting from a state $\mathrm{w}=\left(z_{-n+1}, \ldots, z_{0}\right)$ in $\mathrm{W}_{n}$, for some $n \geq 0$, the walker moves one step in direction $z$ to state $T_{z} \mathrm{~W}$. This happens with probability $q(\mathrm{w}, z)$. For example, in the case of RWRE,

$$
q(\mathrm{w}, z)=\mathbb{E}^{\mathbb{P}}\left(\frac{\prod_{x, y} \pi_{x, x+y}^{n_{x y}(\mathrm{w})}(\omega)}{\mathbb{E}^{\mathbb{P}}\left(\prod_{x, y} \pi_{x, x+y}^{n_{x y}(\mathrm{w})}\right)} \pi_{0 z}(\omega)\right) .
$$

This is well-defined, since only a finite number of $x$ 's is involved. The process $Q_{\phi}$, starting with no history, corresponds to $P_{0}$. Notice that one can also consider $Q_{\mathrm{w}}$ as generating a random walk $\left(X_{n}\right)_{n \geq 1}$ in the future, with increments $\left(Z_{n}=\right.$ $\left.X_{n}-X_{n-1}\right)_{n \geq 1}$ and past $\mathrm{w}$. The connection between these two points of view is seen through the map $z_{j}=z_{0}\left(\mathrm{w}_{j}\right)$. We will still denote this process by $Q_{\mathrm{w}}$. In order to allow this transient Markov chain to start from w in any larger space, we need to extend $q$ to be defined on that space. If $\mathbb{P}$ is a product measure, in the case of a RWRE, then

$$
q(\mathrm{w}, z)=\mathbb{E}^{\mathbb{P}}\left(\frac{\prod_{y} \pi_{0 y}^{n_{0 y}(\mathrm{w})}(\omega)}{\mathbb{E}^{\mathbb{P}}\left(\prod_{y} \pi_{0 y}^{n_{0 y}(\mathrm{w})}\right)} \pi_{0 z}(\omega)\right),
$$

and the same formula would be valid on $\mathrm{W}_{\infty}$ whenever

$$
\lim _{j \rightarrow-\infty}\left|x_{j}(\mathrm{w})\right|=\infty \text {. }
$$

The subspace of such transient walks will be denoted by $\mathrm{W}_{\infty}^{\mathrm{tr}}$, and we will use $\mathrm{W}^{\mathrm{tr}}=\bigcup_{n \geq 0} \mathrm{~W}_{n} \cup \mathrm{W}_{\infty}^{\mathrm{tr}}$. If $\mathbb{P}$ is not a product measure or if we are dealing with a more general walk, then one needs to address the question of defining $q(\mathrm{w}, z)$ for $\mathrm{w} \in \mathrm{W}_{\infty}^{\mathrm{tr}}$ and $|z| \leq M$.

If we view $\mathrm{W}$ as a subspace of $W_{0} \cup\{S, e:|e| \leq M\}^{-\mathbb{N}}$ with the product topology, then it is compact, and $x_{j}$ is continuous for all $j \leq 0$. However, $n_{x z}$, and consequently $q(\mathrm{w}, z)$, will not be continuous, even when $\infty$ is added to compactify the set of integers. To this end, one can always find a metric inducing a topology on $\mathrm{W}$ that keeps the $x_{j}$ 's continuous but also makes the $n_{x z}$ 's, as functions from $\mathrm{W}$ into $\overline{\mathbb{N}}=\{0,1, \ldots, \infty\}$ with another suitable metric, continuous as well. Notice that, in the i.i.d. case, the continuity of the $n_{x z}$ 's under this new topology makes $q(\cdot, z)$ 
continuous on $\mathrm{W}^{\mathrm{tr}}$. This is not always the case, once we do not have a product environment. Thus, in Theorem 2.1 below, this continuity property becomes part of the assumptions and has to be checked later in Theorems 3.3 and 5.1 when Theorem 2.1 is invoked.

In any case, $\mathrm{W}$ is no longer compact for the new topology. However, using the Stone-Čech compactification theorem, one can compactify W, guaranteeing, at the same time, the existence of continuous extensions to the $x_{j}$ 's, $n_{x z}$ 's, $q(\cdot, z)$ 's, $T_{z}$ 's, and $T^{*}$. This is discussed in more detail in the proof of Theorem 2.1 below. Let us denote this compactification of $\mathrm{W}$ by $\overline{\mathrm{W}}$.

Let $\overline{\mathcal{I}}$ be the set of $T^{*}$-invariant measures on $\overline{\mathrm{W}}$, and $\overline{\mathcal{E}}$ the set of ergodic ones. Similarly, let $\mathcal{I}$ (respectively, $\mathcal{E}$ ) be the $T^{*}$-invariant (respectively, ergodic) measures on $\mathrm{W}$. Now that one has a Feller process on the compact space $\overline{\mathrm{W}}$, one can use the standard large-deviations theory for the empirical measures

$$
\mathcal{R}_{n}=n^{-1} \sum_{j=0}^{n} \delta_{T_{Z_{j}} \cdots T_{Z_{1}} \phi} .
$$

The rate function in this case is given by

$$
\mathcal{J}(\mu)=\sup _{u \in \mathcal{C}^{+}(\overline{\mathrm{W}})} \int \log \frac{u(\mathrm{w})}{(q u)(\mathrm{w})} \mu(d \mathrm{w}),
$$

where $\mathcal{C}^{+}(\overline{\mathrm{W}})$ is the set of positive continuous functions on $\overline{\mathrm{W}}$, and

$$
q u(\mathrm{w})=\sum_{z} q(\mathrm{w}, z) u\left(T_{z} \mathrm{w}\right) .
$$

Note, however, that, unless one has a transitivity condition, one can only have, for $G$ open,

$$
\varliminf_{n \rightarrow \infty} n^{-1} \sup _{\mathrm{w}} \log Q_{\mathrm{w}}\left(\mathcal{R}_{n} \in G\right) \geq-\inf _{\mu \in G \cap \mathcal{E}} \mathcal{J}(\mu)
$$

see, for example, [3, 7]. This is useless in our case, and we will instead use the ergodic theorem to prove the lower bound that we need.

On the other hand, because of the way $q$ is defined, $\mathcal{J}(\mu)$ is also the relative entropy of the stationary process $\left(Z_{n}\right)_{n \geq 1}$ that $\mu$ generates, with conditional transitions $\hat{q}_{\mu}(\mathrm{w}, z)=E^{\mu}\left(Z_{1}=z \mid \mathrm{w}\right)$ with respect to the Markov process $Q_{\phi}$. This does not need any transitivity condition and depends only on compactness and the Feller property; see [3, 7].

However, since distinct ergodic measures have disjoint supports, one can make $\hat{q}_{\mu}$ universal over the ergodic ones, that is, independent of $\mu$. Thus,

$$
\mathcal{J}(\mu)=\int \sum_{z} \hat{q}(\mathrm{w}, z) \log \frac{\hat{q}(\mathrm{w}, z)}{q(\mathrm{w}, z)} d \mu(\mathrm{w}) .
$$

The rate function $\mathcal{J}$ is then linear over $T^{*}$-invariant measures. Notice that any $\mu \in \overline{\mathcal{I}}$ generates a stationary process $\left(Z_{n}\right)_{n \in \mathbb{Z}}$, and one can define its mean to be

$$
m(\mu)=E^{\mu}\left(Z_{0}\right)=E^{\mu}\left(-X_{-1}\right) .
$$


The contraction principle suggests then the rate function for the position $X_{n}$ to be, for $\xi \neq 0$,

$$
H(\xi)=\inf _{\substack{\mu \in \mathcal{E} \\ m(\mu)=\xi}} \mathcal{J}(\mu) .
$$

Note that the above infimum runs over measures in $\mathcal{E}$, not $\overline{\mathcal{E}}$.

To state our next theorem, we will need the following notation: For $\ell \in \mathbb{S}^{d-1}$ and $\Lambda \subset \mathbb{Z}^{d}$ finite, connected, and with $0 \in \Lambda$, let

$$
\begin{aligned}
\mathrm{W}_{n}^{\ell,-} & =\left\{\mathrm{w} \in \mathrm{W}_{n}: n_{x}(\mathrm{w})=0 \text { for } x \cdot \ell>0\right\}, \\
\mathrm{W}_{n}^{\Lambda} & =\left\{\mathrm{w} \in \mathrm{W}_{n}: z_{-n+1}, \ldots, z_{-n+1}+\cdots+z_{0} \in \Lambda\right\}, \\
\mathrm{W}^{\ell} & =\left\{\mathrm{w} \in \mathrm{W}_{\infty}^{\mathrm{tr}}: z_{i} \cdot \ell>0 \text { for } i \leq 0\right\} .
\end{aligned}
$$

Also, for $\mathrm{w}=\left(z_{j}\right)_{-n<j \leq 0} \in \mathrm{W}_{n}$, define $T_{\mathrm{w}}=T_{z_{-n+1}} \cdots T_{z_{0}}$, and for $\mathrm{w}=\left(z_{j}\right)_{j \leq 0} \in$ $\mathrm{W}_{\infty}$, define $\mathrm{w}^{(n)}=\left(z_{j}\right)_{-n<j \leq 0} \in \mathrm{W}_{n}$. For a finite connected set $C \subset \mathbb{Z}^{d}$ containing 0 and $\mathrm{w} \in \bigcup_{n \geq 0} \mathrm{~W}_{n}$, define

$$
\sigma_{C, \mathrm{w}}=-\varliminf_{n \rightarrow \infty} n^{-1} \log Q_{\mathrm{w}}\left(X_{j} \in C, 1 \leq j \leq n\right) .
$$

Finally, define the number

$$
H(0)=-\log \inf _{\theta \in \mathbb{R}^{d}} \sup _{p \in \mathcal{K}} \sum_{z} e^{\theta \cdot z} p(z),
$$

where $\overline{\mathcal{K}}$ is the closure of the convex hull of the set of transitions $\{q(\mathrm{w}, \cdot): \mathrm{w} \in$ $\overline{\mathrm{W}}$ \}. One then has the following theorem:

THEOREM 2.1 Let $q$ be a transition probability for a random walk on $\mathbb{Z}^{d}$ satisfying the regularity hypothesis A'. Assume also that the following five requirements are met:

(i) There exists a function $\bar{q}(\mathrm{w}, z)$, defined for all $\mathrm{w} \in \mathrm{W}^{\mathrm{tr}}$ and $z$ with $|z| \leq M$, such that $\bar{q}$ coincides with $q$ on $\bigcup_{n} \mathrm{~W}_{n}$. We will still use the notation $q$ instead of $\bar{q}$.

(ii) For all $z$ fixed with $|z| \leq M, q(\cdot, z)$ as defined above is continuous for the topology on $\mathrm{W}$, restricted to $\mathrm{W}^{\mathrm{tr}}$, that makes the $x_{j}$ 's and $n_{x z}$ 's continuous.

(iii) There exists a function $H(n, S, \mathcal{Z})$, for each $n \geq 0, S \subset \mathbb{Z}^{d}$, and $\mathcal{Z}=$ $\left(z_{i}\right)_{i \geq 0}$, such that

(a) $H(n, S, \mathcal{Z})$ depends only on $z_{1}, \ldots, z_{n}$.

(b) If $\lim _{n \rightarrow \infty} n^{-1}\left(z_{1}+\cdots+z_{n}\right)=\xi \neq 0$ and $\sup _{x \in S} x \cdot \ell<\infty$ for some $\ell \in \mathbb{S}^{d-1}$ with $\xi \cdot \ell>0$, then $\sup _{n} H(n, S, \mathcal{Z})<\infty$.

(c) There exists a constant $C$ such that, for $\mathrm{w}_{1}, \mathrm{w}_{2} \in \mathrm{W}^{\mathrm{tr}}$, one has

$$
\left|\log \frac{d Q_{\mathrm{w}_{1}}}{d Q_{\mathrm{w}_{2}}}\right|_{\mathcal{F}_{n}}(\mathcal{Z}) \mid \leq C H\left(n, S\left(\mathrm{w}_{1}\right) \cup S\left(\mathrm{w}_{2}\right), \mathcal{Z}\right),
$$

where $\mathcal{F}_{n}$ is the $\sigma$-field generated by $Z_{1}, \ldots, Z_{n}$, and $S(\mathrm{w})=\{x$ : $\left.n_{x}(\mathrm{w})>0\right\}$. 
(iv) The following condition is satisfied, for every $\ell \in \mathbb{S}^{d-1}, \mathrm{w}_{2} \in \mathrm{W}^{\ell}$, and $\Lambda \subset\left\{x \in \mathbb{Z}^{d}: x \cdot \ell \geq 0\right\}$ finite, connected, and containing 0 :

$$
\lim _{A \rightarrow \infty} \sup _{\substack{n, m \geq 1 \\|z| \leq M}} \sup _{\substack{\mathrm{w}_{1} \in \mathrm{W}_{n}^{\Lambda} \\ \mathrm{w}_{3} \in \mathrm{W}_{m}^{\ell,-}}}\left|\log \frac{q\left(T_{\mathrm{w}_{1}} T_{\mathrm{w}_{2}^{(A)}} \mathrm{W}_{3}, z\right)}{q\left(T_{\mathrm{w}_{1}} \mathrm{w}_{2}, z\right)}\right|=0 .
$$

(v) For any sequence $\left(C_{L}\right)$ of finite, connected subsets that increase to $\mathbb{Z}^{d}$ and always contain 0 , we have

$$
\sup _{\mathrm{w} \in \bigcup_{n \geq 0} \inf _{n}} \sigma_{C_{L}, \mathrm{w}} \leq H(0)
$$

where $\sigma_{C, \mathrm{w}}$ and $H(0)$ are defined in (2.6) and (2.7) above.

Then the random walk satisfies a large-deviations principle for the position, with a convex, lower-semicontinuous rate function $H$, continuous at 0 , and given by (2.5), for $\xi \neq 0$ and by (2.7) for $\xi=0$.

REMARK 2.2 This theorem mainly reformulates the result of Varadhan [8] for the case of more general random walks. The only novelty in our theorem is the introduction of only five requirements that need to be checked to extend Varadhan's proof and result to a wider class of applications. If one uses $H(n, S, \mathcal{Z})=$ $\sum_{j=0}^{n} \mathbb{I}_{S}\left(x_{j}\right)$, then these requirements are automatically satisfied, in [8], due to the i.i.d. assumption on the environment. They become part of the hypotheses in the more general case. Having done that, the proof of [8] still applies. Nevertheless, we will sketch it to point out where the five requirements come into play.

REMARK 2.3 One notices that the first four requirements are direct conditions on $q$, while the fifth requirement is not. We believe that (v) follows from (i)-(iv). However, we do not prove that in this paper. Instead, we show that it is satisfied in all the examples we consider. In fact, condition (v) says that the annealed rate at 0 is no larger than the quenched one. This is, of course, a known fact in the case of random walks in random ergodic environments, due to Jensen's inequality.

REMARK 2.4 Due to the last theorem in [8, theorem 8.1], one also knows that the set of zeros of $H$ is either a single point or a line segment containing 0 . Moreover, at each extreme $\xi \neq 0$ of this set, one has a unique measure $\alpha$ that is invariant and ergodic for $q$ with $m(\mu)=\xi$. The proof is independent of the i.i.d. assumption and works as long as (2.8) is satisfied.

REMARK 2.5 The min-max representation of $H(0)$ in (2.7) implies that $H(0)=0$ if and only if one has nestling; that is, 0 is in the range of the drift $\left\{D=\sum_{z} z p(z)\right.$ : $p \in \overline{\mathcal{K}}$ \}. In the case of random walks in a random environment, this set is the same as the closure of the convex hull of the support of the drift $D(\omega)=E_{0}^{\omega}\left(X_{1}\right)$; see Remark 5.2 below.

PROOF: The first hypothesis of the theorem is needed to define the process $Q_{\mathrm{w}}$. The second hypothesis makes it a Feller process on a larger space $\overline{\mathrm{W}}$. Indeed, 
as mentioned above, one can always choose a topology that makes the $x_{j}$ 's and $n_{x z}$ 's continuous. The shifts $T^{*}$ and $T_{z}$ are thus continuous as well. Furthermore, hypothesis (ii) then says that $q(\cdot, z)$ is continuous on $\mathrm{W}^{\mathrm{tr}}$ under this new topology. Considering the countable family $\left\{x_{j}, n_{x z}, q(\cdot, z), T_{z}, T^{*}\right\}$ of continuous functions on $\mathrm{W}^{\mathrm{tr}}$, one can use the Stone-Čech compactification theorem (see, for example, [4, theorem 8.2]) to compactify $\mathrm{W}^{\mathrm{tr}}$, which is dense in $\mathrm{W}$, by embedding it in a larger compact space $\overline{\mathrm{W}}$, so that $\mathrm{W}$ is also densely embedded in $\overline{\mathrm{W}}$ and the above functions admit continuous extensions. Of course, for $T^{*}$, one has to exclude $\mathrm{W}_{0}$, since it does not operate on that space.

For the lower bound, it is enough to consider open balls around some $\xi \neq 0$ and obtain a lower bound for $Q_{\phi}\left(\left|n^{-1} X_{n}-\xi\right|<\varepsilon\right)$. Recall that $Q_{\mathrm{w}}$ generates a process $\left(Z_{n}\right)_{n \geq 1}$, which we will still denote by $Q_{\mathrm{w}}$. Now if $\mu \in \mathcal{E}$ is a measure that forces the velocity to be $\xi$, that is, with $m(\mu)=\xi$, then, since $\xi \neq 0$ and $\mu$ is stationary, the third requirement of Theorem 2.1 allows us to replace $Q_{\phi}$ by $Q_{\mathrm{w}}$, for $\mu$-a.e. w, at no extra cost. But if one starts with a $\mu$-typical w, then the ergodic theorem implies that the price to pay for following the statistics of $\mu$ would be $\mathcal{J}(\mu)$. This provides the lower bound and follows from general principles. For the actual proof, see [8, lemma 7.3].

From (2.7), one immediately deduces the upper bound at 0 . Indeed,

$$
n^{-1} \log P_{0}\left(X_{n}=0\right) \leq \inf _{\theta \in \mathbb{R}^{d}} n^{-1} \log E_{0}\left(e^{\theta \cdot X_{n}}\right) \leq-H(0) .
$$

Next, one shows that $\lim _{\xi \rightarrow 0} H(\xi)=H(0)$. As in lemma 7.2 of [8], one can use (2.3) to show that

$$
H(0) \leq \inf _{\substack{\mu \in \overline{\mathcal{I}} \\ m(\mu)=0}} \mathcal{J}(\mu) .
$$

From this and the lower semicontinuity of $\mathcal{J}$, it immediately follows that $H(0) \leq$ $\underline{\lim }_{\xi \rightarrow 0} H(\xi)$. To show the other direction requires a combinatorial lemma.

LEMMA 2.6 Assuming (2.9) and (2.10) hold, one has

$$
\varlimsup_{\xi \rightarrow 0} H(\xi) \leq H(0) \text {. }
$$

PROOF: Fix $\ell \in \mathbb{S}^{d-1}$ and $\mathrm{w}_{\ell} \in \mathrm{W}^{\ell}$. Let $C_{L} \subset \mathbb{Z}^{d}$ be a connected box of side $L$, with 0 on its boundary, and such that, other than visiting $0, \mathrm{w}_{\ell}$ does not go inside of $C_{L}$. Define, for $m \geq 1, \sigma_{L, m}=\sigma_{C_{L}, \mathrm{w}_{\ell}^{(m)}}$. Then, for $\varepsilon>0, \exists \bar{\mu}=\bar{\mu}_{L, m, \varepsilon} \in \mathcal{E}$ such that

$$
\mathcal{J}_{\mathrm{w}_{\ell}^{(m)}}(\bar{\mu}) \leq \sigma_{L, m}+\varepsilon,
$$

where $\mathcal{J}_{\mathrm{w}}(\mu)$ is the relative entropy of the process generated by $\mu$ to the process $Q_{\mathrm{w}}$. Define $\bar{\beta}$ as the countable product of independent copies of $\bar{v}_{C \delta n} \otimes$ $\bar{\mu}_{n-C \delta n-C L} \times \bar{\gamma}_{C L}$. Here, $\bar{v}_{C \delta n}$ is a Dirac mass over a deterministic path that goes in direction $\ell$ for $C \delta n$ steps, $\bar{\mu}_{n-C \delta n-C L}$ is the marginal of the first $n-C \delta n-C L$ increments under $\bar{\mu}_{L, m, \varepsilon}$, and $\bar{\gamma}_{C L}$, conditioned on knowing $z_{1} \cdots z_{n-C L}$, simply 
brings the walker, in $C L$ steps, back to $z_{1}+\cdots+z_{C \delta n-1}$, closing the loop that it goes through in the last $n-C \delta n$ steps. Finally, define $\bar{\alpha}=\bar{\alpha}_{L, m, n, \delta, \varepsilon, \ell}$ as the $n^{\text {th }}$ Cesaro mean of shifts of $\bar{\beta}$.

Then one can estimate $\mathcal{J}_{\mathrm{w}_{\ell}^{(m)}}(\bar{\alpha})$ as follows: We know that

$$
\mathcal{J}_{\mathrm{w}_{\ell}^{(m)}}(\bar{\alpha})=n^{-1} \sum_{i=0}^{n-1} \int \sum_{z} \hat{q}_{\bar{\alpha}}(\mathrm{w}, z) \log \frac{\hat{q}_{\bar{\alpha}}(\mathrm{w}, z)}{q(\mathrm{w}, z)} d \bar{\beta} \circ\left(T^{*}\right)^{i}(\mathrm{w}) .
$$

By ellipticity, the first $C \delta n$ terms can be bounded by $C \delta \log \kappa^{-1}$. Also, the last $C L$ terms can be bounded by $C L n^{-1} \log \kappa^{-1}$. For $C \delta n \leq i<n-C L, \hat{q}_{\bar{\alpha}}(\mathrm{w}, z)$ can be replaced by $\hat{q}_{\bar{\mu}}\left(\left(z_{1}, \ldots, z_{i-C \delta n}\right), z\right)$. This is because of the product structure of $\bar{\beta}$. One can then replace $q(\mathrm{w}, z)$ first by $q\left(T_{z_{i}-C \delta n} \cdots T_{z_{1}} \mathrm{w}_{\ell}, z\right)$, and then replace the latter by $q\left(T_{z_{i-C \delta n}} \cdots T_{z_{1}} \mathrm{w}_{\ell}^{(m)}, z\right)$. The sum of the terms for $C \delta n \leq i<n-C L$ then becomes

$$
\frac{\left(1-C \delta-C L n^{-1}\right)}{N} \sum_{i=0}^{N-1} \int \sum_{z} \hat{q}_{\bar{\mu}}\left(\left(z_{1}, \ldots, z_{i}\right), z\right) \log \frac{\hat{q}_{\bar{\mu}}\left(\left(z_{1}, \ldots, z_{i}\right), z\right)}{q\left(T_{z_{i}} \cdots T_{z_{1}} \mathrm{w}_{\ell}^{(m)}, z\right)} d \bar{\mu},
$$

where $N=n-C \delta n-C L$. The first replacement induces an error term that depends only on $A=C(\delta n-L)$ and that decays to 0 with $n$, due to the decay condition (2.9). The second replacement induces an error that depends only on $A=m$ and will be denoted by $\epsilon_{m}$. It also decays to 0 with $m$ due to (2.9). By bounded convergence, the above expression converges to $(1-C \delta) \mathcal{J}_{\mathbf{w}_{\ell}^{(m)}}(\bar{\mu})$ as $n$ grows, and, therefore, one has for $n$ large enough

$$
\begin{aligned}
H(\delta \ell) & \leq \mathcal{J}_{\mathrm{w}_{\ell}^{(m)}}(\bar{\alpha}) \\
& \leq C \delta \log \kappa^{-1}+C L n^{-1} \log \kappa^{-1}+\sigma_{L, m}(1-C \delta)+2 \varepsilon(1-C \delta)+\epsilon_{m} .
\end{aligned}
$$

Taking $n$ to infinity and then $\varepsilon$ to 0 , one has

$$
H(\delta \ell) \leq C \delta \log \kappa^{-1}+\sigma_{L, m}(1-C \delta)+\epsilon_{m} .
$$

By (2.10), we have $\inf _{L} \sigma_{L, m} \leq H(0)$. Thus,

$$
H(\delta \ell) \leq C \delta \log \kappa^{-1}+H(0)(1-C \delta)+\epsilon_{m} .
$$

Taking $\delta$ to 0 and $m$ to $\infty$ completes the proof.

The linearity of the rate function in (2.4), along with (2.11), easily yields the convexity of $H$ at 0 . To prove the convexity of $H$ away from 0 , one considers $\mu_{1}, \mu_{2} \in \mathcal{E}$, with $m\left(\mu_{i}\right)=\xi_{i}$ and $\xi_{1}$ and $\xi_{2}$ being in a half-plane not containing 0 . If one manages to approximate the measure $\mu=\theta \mu_{1}+(1-\theta) \mu_{2} \in \mathcal{I}$ by a sequence of measures $\mu_{l} \in \mathcal{E}$, with the same velocity $m\left(\mu_{l}\right)=m(\mu)$, then the 
lower semicontinuity and the linearity of $\mathcal{J}$ imply

$$
\begin{aligned}
H(\xi)=\inf _{\substack{\mu \in \mathcal{E} \\
m(\mu)=\xi}} \mathcal{J}(\mu) & \leq \inf _{\substack{\mu_{1}, \mu_{2} \in \mathcal{E} \\
m\left(\mu_{i}\right)=\xi_{i}, l \geq 1}} \mathcal{J}\left(\mu_{l}\right) \\
& \leq \inf _{\substack{\mu_{1}, \mu_{2} \in \mathcal{E} \\
m\left(\mu_{i}\right)=\xi_{i}}}\left(\theta \mathcal{J}\left(\mu_{1}\right)+(1-\theta) \mathcal{J}\left(\mu_{2}\right)\right) \\
& \leq \theta H\left(\xi_{1}\right)+(1-\theta) H\left(\xi_{2}\right)
\end{aligned}
$$

It is always possible to approximate $\mu$ using averages of $l$ translates of product measures over distinct blocs of length $l$. The difficulty is to show that $\mu_{l}$ converges to $\mu$ rather than to some other $\bar{\mu} \in \overline{\mathcal{I}}$. This is where one needs to show that the compactification $\overline{\mathrm{W}}$ is only for convenience and does not affect things much. Indeed, one can show that any measure $\bar{\mu} \in \overline{\mathcal{I}}$ in the set of limit points of $\left(\mathcal{R}_{n}\right)_{n \geq 1}$, with $m(\bar{\mu})=\xi \neq 0$, can be written as $\bar{\mu}=\bar{v}_{1}+\bar{v}_{2}$, with $\bar{v}_{1} \in \mathcal{I}$ and $m\left(\bar{v}_{1}\right)=\xi$. The proof of this fact is another combinatorial lemma that essentially constructs $\bar{v}_{1}$ as the outcome of considering only the runs during which the walker does not backtrack "too much," making $\bar{v}_{1}\left(\mathrm{~W}_{\infty}^{\mathrm{tr}}\right)=1$. This construction is independent of whether $\mathbb{P}$ is a product measure and is done in $[8, \mathrm{sec} .6$, theorem 6.1$]$. On the other hand, the measure $\bar{\mu}$ will then be represented by measures in $\overline{\mathcal{E}}$ with velocities $\xi_{1}$ or $\xi_{2}$, and therefore so will be $\bar{v}_{2}$. But since $m\left(\bar{v}_{2}\right)=0$ is not on the segment joining these two vectors, $\bar{v}_{2}=0$ and $\bar{\mu}=\mu$, leading to the convexity of $H$ away from 0 .

Finally, the upper bound, away from 0 , follows easily from the large deviations for $\left(\mathcal{R}_{n}\right)$, along with the above decomposition of $\bar{\mu}$, the linearity of $\mathcal{J}$, inequality (2.11), and the convexity of $H$. See the proof of theorem 3.1 in [8] for the details.

\section{Large Deviations for Reinforced Random Walks: Two Examples}

Consider a random walk given by

$$
q_{e}(\mathrm{w}, z)=\frac{n_{0 z}^{N}+1}{\sum_{|\bar{z}| \leq M}\left(n_{0 \bar{z}}^{N}+1\right)}
$$

for $|z| \leq M$ and $\mathrm{w} \in \mathrm{W}^{\mathrm{tr}}$. Here $n_{x y}^{N}=\min \left(n_{x y}, N\right)$. Such a walk is called the $N$-times edge-reinforced random walk. Another model is the $N$-times vertexreinforced random walk. In this case we have, for $|z| \leq M$ and $\mathrm{w} \in \mathrm{W}^{\mathrm{tr}}$,

$$
q_{v}(\mathrm{w}, z)=\frac{n_{z}^{N}+1}{\sum_{|\bar{z}| \leq M}\left(n_{\bar{z}}^{N}+1\right)}
$$

where $n_{x}^{N}=\min \left(n_{x}, N\right)$.

REMARK 3.1 Usually, one takes $M=1$ and does not include $z=0$ in the definition of the above transition rules. This is not, of course, mandatory. 
Clearly, these two models satisfy hypothesis $\mathrm{A}^{\prime}$ as well as the first two requirements of Theorem 2.1. The third requirement of the theorem is also clearly satisfied, due to ellipticity, with $H(n, S, \mathcal{Z})=\sum_{j=0}^{n} \mathbb{I}_{S_{M}}\left(x_{j}\right)$, where $S_{M}=\left\{x \in \mathbb{Z}^{d}\right.$ : $\operatorname{dist}(x, S) \leq M\}$ and $M$ is the range of the increments. The fourth requirement is satisfied due to the local nature of the models we are considering. In fact, the term in (2.9) vanishes as soon as $A>M$. One also has the following lemma:

LEMMA 3.2 Condition (2.10) holds.

ProOF: Let $C_{L}$ be a sequence of finite connected subsets, all containing 0 and increasing to $\mathbb{Z}^{d}$.

First, notice that $H(0)=0$, since uniform transitions clearly belong to $\overline{\mathcal{K}}$. Second, notice that, by standard arguments, as in [8, sec.7], one has

$$
\begin{aligned}
& \inf _{L}\left(-\varliminf_{n \rightarrow \infty} n^{-1} \log \tilde{P}_{0}\left(X_{j} \in C_{L}, 1 \leq j \leq n\right)\right) \leq \\
& \lim _{n \rightarrow \infty}(p n)^{-1} \log \tilde{P}_{0}\left(X_{p n}=0\right)=0,
\end{aligned}
$$

where $p$ is the period of $\tilde{P}_{0}$ and $\tilde{P}_{0}$ is the symmetric random walk on $\mathbb{Z}^{d}$ that jumps from each site $x$, with equal probability, to one of the sites in $\left\{x+z: p_{0}(z)>0\right\}$. See also Lemma 5.5 for a sketch of the proof of (3.1)-like statements.

Now, take $\gamma>0$, and let $L$ be such that

$$
-\underline{\lim }_{n \rightarrow \infty} n^{-1} \log \tilde{P}_{0}\left(X_{j} \in C_{L}, 1 \leq j \leq n\right) \leq \gamma .
$$

Then, for this fixed $L$, the walk under $Q_{\mathrm{w}}$, with finite length history w, can first fill out $C_{L}$, visiting all sites and edges at least $N$ times and returning to 0 . This will take only $C_{d, N} L$ steps, with $C_{d, N}$ being some constant that depends on $d$ and $N$ only. Due to ellipticity, this procedure will not affect $\sigma_{C_{L} \text {,w }}$. But now, inside $C_{L}$, the law of $Q_{\mathrm{w}}$ is the same as $\tilde{P}_{0}$. This shows that $\sigma_{C_{L}, \mathrm{w}} \leq \gamma$ for all w. Taking $L$ to infinity and then $\gamma$ to 0 finishes the proof.

The following theorem is then a corollary of Theorem 2.1 .

THEOREM 3.3 Both the $N$-times edge-and vertex-reinforced random walks satisfy a large-deviations principle for the position. Moreover, the only zero of the rate function is at 0 .

PROOF: The proof of the statement about the zeros of the rate function follows from Remark 2.4. Indeed, as we mentioned in Remark 2.4, the set of zeros of the rate function is either a single point or a segment passing through 0 . But since both models are isotropic, this set has to be the singleton $\{0\}$.

One has the following consequence of the above theorem:

COROLLARY 3.4 Both the $N$-times edge-and vertex-reinforced random walks satisfy a law of large numbers with zero velocity:

$$
P_{0}\left(n^{-1} X_{n} \rightarrow 0\right)=1 .
$$




\section{Mixing Random Environments}

We now switch back to random walks in mixing random environments. First, we introduce some notation. For a set $V \subset \mathbb{Z}^{d}$, let us denote by $\Omega_{V}$ the set of possible configurations $\omega_{V}=\left(\omega_{x}\right)_{x \in V}$ and by $\mathfrak{S}_{V}$ the $\sigma$-field generated by the environments $\left(\omega_{x}\right)_{x \in V}$. For a probability measure $\mathbb{P}$, we will denote by $\mathbb{P}_{V}$ the projection of $\mathbb{P}$ onto $\left(\Omega_{V}, \mathfrak{S}_{V}\right)$. For $\omega \in \Omega$, we will denote by $\mathbb{P}_{V}^{\omega}$ the regular conditional probability, knowing $\mathfrak{S}_{\mathbb{Z}^{d}-V}$ on $\left(\Omega_{V}, \mathfrak{S}_{V}\right)$. Furthermore, for $\Lambda \subset V, \mathbb{P}_{V, \Lambda}^{\omega}$ will denote the projection of $\mathbb{P}_{V}^{\omega}$ onto $\left(\Omega_{\Lambda}, \mathfrak{S}_{\Lambda}\right)$. Also, we will use the notation $V^{c}=\mathbb{Z}^{d}-V, \partial_{r} V=\left\{x \in \mathbb{Z}^{d}-V: \operatorname{dist}(x, V) \leq r\right\}$ with $r \geq 0$, and $|V|$ will denote the cardinality of $V$. Finally, for $\omega, \bar{\omega} \in \Omega, V, W \subset \mathbb{Z}^{d}$ with $V \cap W=\varnothing$, we will use $\left(\bar{\omega}_{V}, \omega_{W}\right)$ to denote $\overline{\bar{\omega}}_{V \cup W}$ such that $\overline{\bar{\omega}}_{V}=\bar{\omega}_{V}$ and $\overline{\bar{\omega}}_{W}=\omega_{W}$.

Consider a reference product measure $\alpha$ on $(\Omega, \mathfrak{S})$ and a family of functions $U=\left(U_{A}\right)_{A \subset \mathbb{Z}^{d}}$, called an interaction, such that $U_{A} \equiv 0$ if $|A|>r$ (finite range), $U_{A}(\omega)$ depends only on $\omega_{A}, \beta=\sup _{A, \omega}\left|U_{A}(\omega)\right|<\infty$ (bounded interaction), and $U_{\theta^{x} A}\left(\theta^{x} \omega\right)=U_{A}(\omega)$ (shift invariant). One can then define the specification

$$
\frac{d \mathbb{P}_{V}^{\omega_{V c}}}{d \alpha_{V}}\left(\omega_{V}\right)=\frac{e^{-H_{V}\left(\omega_{V} \mid \omega_{V c}\right)}}{Z_{V}\left(\omega_{V^{c}}\right)},
$$

where

$$
Z_{V}\left(\omega_{V^{c}}\right)=\mathbb{E}^{\alpha}\left(e^{-H_{V}\left(\omega_{V} \mid \omega_{V c}\right)}\right)
$$

is the partition function and

$$
H_{V}\left(\omega_{V} \mid \omega_{V^{c}}\right)=\sum_{A: A \cap V \neq \phi} U_{A}(\omega)
$$

is the conditional Hamiltonian. The parameter $\beta>0$ is called the inverse temperature. One can ask whether this system of conditional probabilities arises from a probability measure and whether such a measure is unique. In [2], the authors introduce a sufficient condition for this to happen. The Dobrushin-Shlosman strongdecay property holds if there exist $G, g>0$ such that for all $\Lambda \subset V \subset \mathbb{Z}^{d}$ finite, $x \in \partial_{r} V$, and $\omega, \bar{\omega} \in \Omega$, with $\omega_{y}=\bar{\omega}_{y}$ when $y \neq x$, we have

$$
\operatorname{Var}\left(\mathbb{P}_{V, \Lambda}^{\omega}, \mathbb{P}_{V, \Lambda}^{\bar{\omega}}\right) \leq G e^{-g \operatorname{dist}(x, \Lambda)},
$$

where $\operatorname{Var}(\cdot, \cdot)$ is the variational distance $\operatorname{Var}(\mu, v)=\sup _{E \in \mathfrak{S}}(\mu(E)-v(E))$. If the above condition holds, then there exists a unique $\mathbb{P}$ that is consistent with the specification $\left(\mathbb{P}_{V}^{\omega_{V c} c}\right)$. Moreover, we have, for all $\omega \in \Omega$,

$$
\lim _{\operatorname{dist}\left(\Lambda, V^{c}\right) \rightarrow \infty} \operatorname{Var}\left(\mathbb{P}_{V, \Lambda}^{\omega}, \mathbb{P}_{\Lambda}\right)=0 .
$$

If the interaction is translation-invariant and the specification satisfies (4.1), then the unique field $\mathbb{P}$ is also shift-invariant; see [5, sec. 5.2].

One should note that (4.1) is satisfied for several classes of Gibbs fields. In particular, in the high-temperature region (that is, when $\beta$ is small enough; class $\mathcal{A}$ in [1]), in the case of a large magnetic field (class $\mathcal{B}$ in [1]), and in the case of one-dimensional and almost one-dimensional interactions (class $\mathcal{E}$ in [1]); see [1, 
theorem 2.2] for the proof and for the precise definitions of the above classes. It is worthwhile to note that these classes are closed under perturbations by any 0-range interaction. This will be our second condition on the environment $\mathbb{P}$.

Hypothesis B The probability measure $\mathbb{P}$ is the unique Gibbs field corresponding to a finite range interaction such that any perturbation of it by a 0-range interaction $\phi$ satisfies (4.1), with constants $G(\phi)$ and $g(\phi)$.

We are now ready to address the first two requirements of Theorem 2.1 . Let $\mathbb{P}$ be the unique Gibbs field corresponding to a given translation-invariant interaction $U$ of finite range $r$. For $\mathrm{w} \in \mathrm{W}_{\infty}^{\mathrm{tr}}$, define the new interaction $U^{\mathrm{w}}$ such that

$$
U_{A}^{\mathrm{w}}(\omega)= \begin{cases}U_{A}(\omega) & \text { if }|A| \neq 1 \\ U_{\{x\}}(\omega)-\phi_{x}^{\mathrm{w}}(\omega) & \text { if } A=\{x\}\end{cases}
$$

where $\phi_{x}^{\mathrm{w}}(\omega)=\sum_{z} n_{x z}(\mathrm{w}) \log \pi_{x, x+z}(\omega)$, which is bounded, due to hypothesis A. If $U$ satisfies hypothesis $\mathrm{B}$, then $U^{\mathrm{w}}$ satisfies (4.1), and one has a unique Gibbs field $\mathbb{P}^{\mathrm{w}}$ corresponding to $U^{\mathrm{w}}$. We will use $H_{V}^{\mathrm{w}}$ for the conditional Hamiltonian of the partition $U^{\mathrm{w}}$. Let $\bar{q}(\mathrm{w}, z)=\mathbb{E}^{\mathbb{P}^{\mathrm{w}}}\left(\pi_{0 z}\right)$.

LEMMA 4.1 The transition probability $\bar{q}$ is well-defined for $\mathrm{w} \in \mathrm{W}^{\mathrm{tr}}$. Moreover, $\bar{q}$ coincides with q, defined in (2.2), on $\bigcup_{n} \mathrm{~W}_{n}$.

PROOF: The first part of the lemma is trivial. Furthermore, by (2.2), one has, for a fixed $\mathrm{w} \in \bigcup_{n} \mathrm{~W}_{n}$,

$$
\begin{aligned}
& q(\mathrm{w}, z)=\mathbb{E}^{\mathbb{P}}\left(\frac{\prod_{x, y} \pi_{x, x+y}^{n_{x y}(\mathrm{w})}(\omega)}{\mathbb{E}^{\mathbb{P}}\left(\prod_{x, y} \pi_{x, x+y}^{n_{x y}(\mathrm{w})}\right)} \pi_{0 z}(\omega)\right) \\
& =\lim _{V \uparrow \mathbb{Z}^{d}} \mathbb{E}^{\alpha}\left(\frac{e^{-H_{V}\left(\omega_{V} \mid \omega_{V c}\right)}}{Z_{V}\left(\omega_{V^{c}}\right)} \frac{e^{\sum_{x \in V} \phi_{x}^{\mathrm{w}}(\omega)}}{\mathbb{E}^{\mathbb{P}_{V}^{\omega_{V}}}\left(e^{\sum_{x \in V} \phi_{x}^{\mathrm{w}}}\right)} \pi_{0 z}(\omega)\right) \\
& =\lim _{V \uparrow \mathbb{Z}^{d}} \mathbb{E}^{\alpha}\left(\frac{e^{-H_{V}\left(\omega_{V} \mid \omega_{V} c\right)} e^{\sum_{x \in V} \phi_{x}^{\mathrm{w}}(\omega)}}{\mathbb{E}^{\alpha}\left(e^{-H_{V}\left(\cdot \mid \omega_{V}\right)} e^{\left.\sum_{x \in V} \phi_{x}^{\mathrm{w}}\right)}\right.} \pi_{0 z}(\omega)\right) \\
& =\lim _{V \uparrow \mathbb{Z}^{d}} \mathbb{E}^{\alpha}\left(\frac{e^{-H_{V}^{\mathrm{w}}\left(\omega_{V} \mid \omega_{V^{c}}\right)}}{\mathbb{E}^{\alpha}\left(e^{-H_{V}^{\mathrm{w}}\left(\cdot \mid \omega_{V^{c}}\right)}\right)} \pi_{0 z}(\omega)\right) \\
& =\lim _{V \uparrow \mathbb{Z}^{d}} \mathbb{E}^{\left(\mathbb{P}^{\mathrm{w}}\right)_{V}^{\omega_{V} c}}\left(\pi_{0 z}\right)=\bar{q}(\mathrm{w}, z) \text {. }
\end{aligned}
$$

We will keep using the notation $q$ instead of $\bar{q}$. Next, we address the question of continuity of $q(\cdot, z)$.

LEMMA 4.2 Consider the topology on $\mathrm{W}$ that makes the $x_{j}$ 's and $n_{x z}$ 's continuous and restrict it to $\mathrm{W}^{\mathrm{tr}}$. Then $q(\cdot, z)$, defined in Lemma 4.1, is continuous for all $z$ with $|z| \leq M$. Moreover, if $Q_{\mathrm{w}}$ is the process of increments $\left(Z_{n}\right)_{n \geq 1}$, starting with 
history $\mathrm{w} \in \mathrm{W}^{\mathrm{tr}}$ and defined using transitions $q$, then we have, for $z_{1}, \ldots, z_{n} \in \mathbb{Z}^{d}$ and $\mathrm{w} \in \mathrm{W}^{\mathrm{tr}}$,

$$
\begin{aligned}
Q_{\mathrm{w}}\left(Z_{1}=z_{1}, \ldots, Z_{n}=z_{n}\right) & =\bar{q}\left(\mathrm{w}, z_{1}\right) \cdots \bar{q}\left(T^{z_{1}+\cdots+z_{n-1}} \mathrm{w}, z_{n}\right) \\
& =\mathbb{E}^{\mathbb{P}^{\mathrm{w}}}\left(\pi_{0 z_{1}} \pi_{z_{1}, z_{1}+z_{2}} \cdots \pi_{z_{1}+\cdots+z_{n-1}, z_{1}+\cdots+z_{n}}\right) .
\end{aligned}
$$

PROOF: Clearly, formula (4.3) is correct for $\mathrm{w} \in \bigcup_{n} \mathrm{~W}_{n}$. Therefore, to prove both statements of our lemma, one only needs to consider a finite $\Lambda \subset \mathbb{Z}^{d}$ and look at

$$
\operatorname{Var}\left(\mathbb{P}_{\Lambda}^{\mathrm{W}}, \mathbb{P}_{\Lambda}^{\overline{\mathrm{W}}}\right) \leq \operatorname{Var}\left(\mathbb{P}_{V, \Lambda}^{\mathrm{W}, \omega_{V^{c}}}, \mathbb{P}_{V, \Lambda}^{\overline{\mathrm{W}}, \omega_{V^{c}}}\right)+\operatorname{Var}\left(\mathbb{P}_{\Lambda}^{\mathrm{W}}, \mathbb{P}_{V, \Lambda}^{\mathrm{W}, \omega_{V^{c}}}\right)+\operatorname{Var}\left(\mathbb{P}_{\Lambda}^{\overline{\mathrm{W}}}, \mathbb{P}_{V, \Lambda}^{\overline{\mathrm{W}}, \omega_{V^{c}}}\right)
$$

Because of the continuity of the $n_{x z}$ 's, one has that, for fixed $\Lambda, V$, and $\omega_{V^{c}}, \mathbb{P}_{V, \Lambda}^{\overline{\mathrm{w}}, \omega_{V^{c}}}$ converges weakly to $\mathbb{P}_{V, \Lambda}^{\mathrm{w}, \omega_{V^{c}}}$, as $\overline{\mathrm{w}}$ converges to $\mathrm{w}$. This has two consequences. On the one hand, this implies that if $\overline{\mathrm{w}}$ is close enough to $\mathrm{w}$, then (4.1) is satisfied for $\mathbb{P}^{\overline{\mathrm{w}}}$ with constants $2 G(\mathrm{w})$ and $g(\mathrm{w})$; see [1, prop. 3.2]. Therefore, inequality (4.2) holds for all $\mathbb{P}^{\overline{\mathrm{w}}}$, with $\overline{\mathrm{w}}$ close enough to $\mathrm{w}$. One can then choose $V$ such that $\operatorname{dist}\left(V^{c}, \Lambda\right)$ is large enough, and the last two terms in the above sum are small, uniformly in $\mathrm{w}$ and $\overline{\mathrm{w}}$. On the other hand, if $\overline{\mathrm{w}}$ is close enough to $\mathrm{w}$, the number of visits to sites inside $V$ will be the same for both walks, and the first term will then vanish. This proves the continuity of $\mathbb{P}^{\mathrm{w}}$ in $\mathrm{w}$, as well as (4.3).

\section{The Large-Deviations Principle for RWRE}

We have the following theorem:

THEOREM 5.1 Let $\mathbb{P}$ satisfy hypotheses $\mathrm{A}$ and $\mathrm{B}$. Then the annealed random walk $P_{0}$ in environment $\mathbb{P}$ satisfies a large-deviations principle for the position, with a convex, lower-semicontinuous rate function $H$ given by (2.5) and (2.7).

REMARK 5.2 Notice that in the case of a random walk in a random environment, the subadditivity of $-\log \pi_{x x}^{(n)}(\omega)$ allows us to write

$$
H(0)=\inf _{n}\left(-n^{-1} \log \pi_{00}^{(n)}(\omega)\right)
$$

for $\omega \in \operatorname{supp}(\mathbb{P})$; see $[8$, sec. 7]. This implies that (2.7) also holds when $\overline{\mathcal{K}}$, the closure of the convex hull of $\{q(\mathrm{w}, \cdot): \mathrm{w} \in \overline{\mathrm{W}}\}$, is replaced by $\hat{\mathcal{K}}$, the closure of the convex hull of the transitions $\left\{\pi_{0, \cdot}(\omega): \omega \in \operatorname{supp}(\mathbb{P})\right\}$; see [8, lemma 7.1].

PROOF: Lemmas 4.1 and 4.2 show that the first two requirements of Theorem 2.1 are satisfied. Thus, we need only the following three lemmas:

LEMMA 5.3 Define $q$ and $Q_{\mathrm{w}}$ as in Lemmas 4.1 and 4.2. Let

$$
H(n, S, \mathcal{Z})=\sum_{i=0}^{n} e^{-g \operatorname{dist}\left(x_{i}, S\right)} .
$$

Then there exists a constant $C$ for which (2.8) holds. 
PROOF: Let $\overline{\mathrm{w}}=\left(z_{1}, \ldots, z_{n}\right), S=\left\{x_{0}, x_{1}, \ldots, x_{n}\right\}$, and $\bar{S}=S-\left(S\left(\mathrm{w}_{1}\right) \cup\right.$ $\left.S\left(\mathrm{w}_{2}\right)\right)$. By (4.3), one has

$$
\left.\frac{d Q_{\mathrm{w}_{1}}}{d Q_{\mathrm{w}_{2}}}\right|_{\mathcal{F}_{n}}(\mathcal{Z})=\frac{\mathbb{E}^{\mathbb{P}^{\mathrm{w}} 1}\left(\prod_{y, x \in \mathbb{S}} \pi_{x, x+y}^{n_{x y}(\overline{\mathrm{w}})}\right)}{\mathbb{E}^{\mathbb{P}^{\mathrm{w}_{2}}}\left(\prod_{y, x \in S} \pi_{x, x+y}^{n_{x y}(\overline{\mathrm{w}})}\right)} .
$$

Due to the ellipticity condition, one can replace $S$ by $\bar{S}$ and bound the missing terms by $C^{\sum_{j=0}^{n} \mathbb{I}_{S\left(\mathrm{w}_{1}\right) \cup S\left(\mathrm{w}_{2}\right)\left(x_{j}\right)}}$ for some deterministic constant $C$. Therefore, we only need to bound, both above and below and uniformly in $V$, the quantity

$$
\frac{\mathbb{E}^{\mathbb{P}_{V, \bar{s}}^{\mathrm{w}_{1}, \omega_{V c}}}\left(\prod_{y, x \in \bar{S}} \pi_{x, x y}^{n_{x y}(\overline{\mathrm{w}})}\right)}{\mathbb{E}^{\mathbb{P}^{\mathrm{w}_{2}, \bar{\omega}}, \overline{\omega_{V} c}}\left(\prod_{y, x \in \bar{S}} \pi_{x, x+y}^{n_{x y}(\overline{\mathrm{w}})}\right)} .
$$

Thus, we are led to bounding $d \mathbb{P}_{V, \bar{S}}^{\mathrm{W}_{1}, \omega_{V c}} / d \mathbb{P}_{V, \bar{S}}^{\mathrm{W}_{2}, \omega_{V c}}$. However,

$$
\begin{aligned}
\frac{d \mathbb{P}_{V, \bar{S}}^{\mathrm{w}_{1}, \omega_{V c}}}{d \mathbb{P}_{V, \bar{S}}^{\mathrm{w}_{2}, \omega_{V c}}} & =\mathbb{E}^{\mathbb{P}_{V}^{\mathrm{w}_{2}, \omega_{V} c}}\left(\frac{d \mathbb{P}_{V}^{\mathrm{w}_{1}, \omega_{V c}}}{d \mathbb{P}_{V}^{\mathrm{w}_{2}, \omega_{V c}}} \mid \mathfrak{S}_{\bar{S}}\right) \\
& =\mathbb{E}^{\mathbb{P}_{V}^{\mathrm{w}_{2}, \omega_{V} c}}\left(\frac{Z_{V}^{\mathrm{w}_{2}}\left(\omega_{V^{c}}\right) e^{-H_{V}^{\mathrm{w}_{1}}\left(\omega_{V} \mid \omega_{V c}\right)}}{Z_{V}^{\mathrm{w}_{1}}\left(\omega_{V c}\right) e^{-H_{V}^{\mathrm{w}_{2}}\left(\omega_{V} \mid \omega_{V c}\right)}} \mid \mathfrak{S}_{\bar{S}}\right) \\
& =\mathbb{E}^{\mathbb{P}_{V}^{\mathrm{w}_{2}, \omega_{V} c}}\left(\frac{\mathbb{E}^{\mathbb{P}_{V}^{\omega_{V} c}}\left(\prod_{y, x \in V} \pi_{x, x+y}^{n_{x y}\left(\mathrm{w}_{2}\right)}\right)}{\mathbb{E}^{\mathbb{P}_{V}^{\omega_{V c}}}\left(\prod_{y, x \in V} \pi_{x, x+y}^{n_{x y}\left(\mathrm{w}_{1}\right)}\right)} \frac{\prod_{y, x \in V} \pi_{x, x+y}^{n_{x y}\left(\mathrm{w}_{1}\right)}\left(\omega_{V}\right)}{\prod_{y, x \in V} \pi_{x, x+y}^{n_{x y}\left(\mathrm{w}_{2}\right)}\left(\omega_{V}\right)} \mid \mathfrak{S}_{\bar{S}}\right) \\
& =\frac{\mathbb{E}^{\mathbb{P}_{V}^{\omega_{V c} c}}\left(\prod_{y, x \in V-\bar{S}} \pi_{x, x+y}^{n_{x, x+y}\left(\mathrm{w}_{2}\right)}\right)}{\mathbb{E}^{\mathbb{P}_{V}^{\omega_{V} c}}\left(\prod_{y, x \in V-\bar{S}} \pi_{x, x+y}^{n_{x y}\left(\mathrm{w}_{1}\right)}\right)} \mathbb{E}^{\mathbb{P}_{V-\bar{S}}^{\mathrm{w}_{2}, \omega_{V c}, \omega_{\bar{S}}}}\left(\frac{\prod_{y, x \in V-\bar{S}} \pi_{x, x+y}^{n_{x y}\left(\mathrm{w}_{1}\right)}}{\prod_{y, x \in V-\bar{S}} \pi_{x, x+y}^{n_{x y}\left(\mathrm{w}_{2}\right)}}\right) .
\end{aligned}
$$

Now, the last expectation in the above series of equalities is equal to

$$
\int \frac{\prod_{y, x \in V-\bar{S}} \pi_{x, x+y}^{n_{x y}\left(\mathrm{w}_{1}\right)}}{\prod_{y, x \in V-\bar{S}} \pi_{x, x+y}^{n_{x y}\left(\mathrm{w}_{2}\right)}} \frac{\prod_{y, x \in V-\bar{S}} \pi_{x, x+y}^{n_{x y}\left(\mathrm{w}_{2}\right)} e^{-H_{V}\left(\omega_{V-\bar{S}} \mid \omega_{V c}, \omega_{\bar{S}}\right)}}{\mathbb{E}^{\mathbb{P}_{V-\bar{S}}^{\omega_{C}, \omega_{\bar{S}}}}\left(\prod_{y, x \in V-\bar{S}} \pi_{x, x+y}^{n_{x y}\left(\mathrm{w}_{2}\right)}\right) Z_{V-\bar{S}}\left(\omega_{V^{c}}, \omega_{\bar{S}}\right)} d \alpha\left(\omega_{V-\bar{S}}\right),
$$

and therefore

$$
\frac{d \mathbb{P}_{V, \bar{S}}^{\mathrm{w}_{1}, \omega_{V^{c}}}}{d \mathbb{P}_{V, \bar{S}}^{\mathrm{w}_{2}, \omega_{V^{c}}}}\left(\omega_{\bar{S}}\right)
$$

is equal to

$$
\frac{\mathbb{E}^{\mathbb{P}_{V}^{\omega_{V} c}}\left(\prod_{y, x \in V-\bar{S}} \pi_{x, y+y}^{n_{x y}\left(\mathrm{w}_{2}\right)}\right)}{\mathbb{E}^{\mathbb{P}_{V}^{\omega_{V} c}}\left(\prod_{y, x \in V-\bar{S}} \pi_{x, x+y}^{n_{x y}\left(\mathrm{w}_{1}\right)}\right)} \frac{\mathbb{E}^{\mathbb{P}_{V-\bar{S}}^{\omega_{V} c, \omega_{\bar{S}}}}\left(\prod_{y, x \in V-\bar{S}} \pi_{x, x+y}^{n_{x y}\left(\mathrm{w}_{1}\right)}\right)}{\mathbb{E}^{\mathbb{P}_{V-\bar{S}}^{\omega_{V} c \omega_{\bar{S}}}}\left(\prod_{y, x \in V-\bar{S}} \pi_{x, x+y}^{n_{x y}\left(\mathrm{w}_{2}\right)}\right)} .
$$


We are then reduced to bounding, for $\mathrm{w} \in\left\{\mathrm{w}_{1}, \mathrm{w}_{2}\right\}$, both above and below and uniformly $\omega \in \Omega$, the term

$$
\frac{\mathbb{E}^{\mathbb{P}_{V}^{\omega} V^{c}}\left(\prod_{y, x \in V-\bar{S}} \pi_{x, x+y}^{n_{x y}(\mathrm{w})}\right)}{\mathbb{E}^{\mathbb{P}_{V-\bar{S}}^{\omega_{V}, \omega}}\left(\prod_{y, x \in V-\bar{S}} \pi_{x, x+y}^{n_{x y}(\mathrm{w})}\right)} .
$$

Noticing that the numerator is an integral of the denominator and that the above product is actually running over the set $V \cap S(\mathrm{w})$, it all boils down to bounding from above the quantity

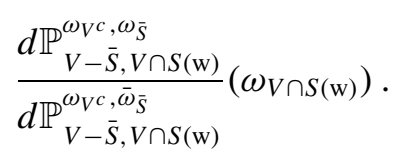

We will do this first for $\omega_{\bar{S}}$ and $\bar{\omega}_{\bar{S}}$ that differ only at a point $x \in \bar{S}$. In this case, we have

$$
\frac{d \mathbb{P}_{V-\bar{S}, V \cap S(\mathrm{w})}^{\omega_{V^{c}}, \omega_{\bar{S}}}}{d \mathbb{P}_{V-\bar{S}, V \cap S(\mathrm{w})}^{\omega_{V^{c}}, \bar{\omega}_{\bar{S}}}}\left(\omega_{V \cap S(\mathrm{w})}\right)=\mathbb{E}^{\mathbb{P}_{V-\bar{S}}^{\omega_{V^{c}}, \bar{\omega}_{\bar{S}}}}\left(\frac{d \mathbb{P}_{V-\bar{S}}^{\omega_{V^{c}}, \omega_{\bar{S}}}}{d \mathbb{P}_{V-\bar{S}}^{\omega_{V^{c}}, \bar{\omega}_{\bar{S}}}} \mid \mathfrak{S}_{V \cap S(\mathrm{w})}\right)\left(\omega_{V \cap S(\mathrm{w})}\right) .
$$

Notice that $F=d \mathbb{P}_{V-\bar{S}}^{\omega_{V^{c}}, \omega_{\bar{S}}} / d \mathbb{P}_{V-\bar{S}}^{\omega_{V^{c}}, \bar{\omega}_{\bar{S}}}$ is bounded by $C_{1}=C_{1}(\beta, r)$, and if we denote by $B(x, r)$ the subset of elements of $\mathbb{Z}^{d}$ within distance $r$ from $x$, then $F$ is $\mathfrak{S}_{(V-\bar{S}) \cap B(x, r)}$-measurable. If, moreover, $B(x, r) \cap S(\mathrm{w})=\varnothing$, then one has

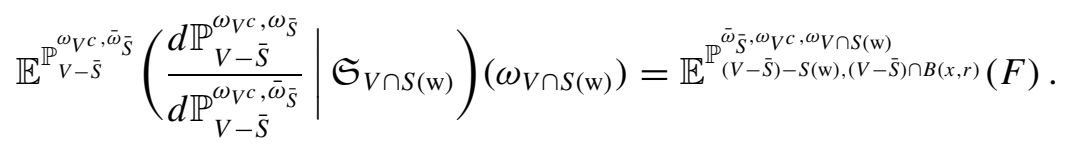

Therefore, using the validity of the Dobrushin-Shlosman condition for our case, one has

$$
\begin{aligned}
& \frac{d \mathbb{P}_{V-\bar{S}, V \cap S(\mathrm{w})}^{\omega_{V^{c}}, \omega_{\bar{S}}}}{d \mathbb{P}_{V-\bar{S}, V \cap S(\mathrm{w})}^{\omega_{V^{c}}, \bar{\omega}_{\bar{S}}}}\left(\omega_{V \cap S(\mathrm{w})}\right)-\frac{d \mathbb{P}_{V-\bar{S}, V \cap S(\mathrm{w})}^{\omega_{V^{c}, \omega_{\bar{S}}}}}{d \mathbb{P}_{V-\bar{S}, V \cap S(\mathrm{w})}^{\omega_{V^{c}}, \bar{\omega}_{\bar{S}}}}\left(\bar{\omega}_{V \cap S(\mathrm{w})}\right) \\
& \leq C_{1} \operatorname{var}\left(\mathbb{P}_{(V-\bar{S})-S(\mathrm{w}),(V-\bar{S}) \cap B(x, r)}^{\bar{\omega}_{\bar{S}}, \omega_{V^{c}}, \omega_{V \cap S(\mathrm{w})}}, \mathbb{P}_{(V-\bar{S})-S(\mathrm{w}),(V-\bar{S}) \cap B(x, r)}^{\bar{\omega}_{\bar{S}}, \omega_{V^{c}}, \bar{\omega}_{V \cap S(\mathrm{w})}}\right) \\
& \leq C_{2} e^{-g \operatorname{dist}(x, S(\mathrm{w}))} .
\end{aligned}
$$

Integrating $\bar{\omega}_{(V-\bar{S}) \cap S(\text { w) }}$ out, one has the upper bound

$$
\frac{d \mathbb{P}_{V-\bar{S},(V-\bar{S}) \cap S(\mathrm{w})}^{\omega_{V^{c}}, \omega_{\bar{S}}}}{d \mathbb{P}_{V-\bar{S},(V-\bar{S}) \cap S(\mathrm{w})}^{\omega_{V^{c}}, \bar{\omega}_{\bar{S}}}} \leq 1+C_{2} e^{-g \operatorname{dist}(x, S(\mathrm{w}))} .
$$

For the case where $B(x, r) \cap S(\mathrm{w}) \neq \varnothing$, we simply use the upper bound $C_{1}$. Then, for a general $\omega_{\bar{S}}$ and $\bar{\omega}_{\bar{S}}$, one has the upper bound to (5.1),

$$
C_{1}^{\operatorname{card}(\{x \in S: B(x, r) \cap S(\mathrm{w}) \neq \varnothing\})} \prod_{x \in S-S(\mathrm{w})}\left(1+C_{2} e^{-g \operatorname{dist}(x, S(\mathrm{w}))}\right) \leq C_{3}^{\sum_{x \in S} e^{-g \operatorname{dist}(x, S(\mathrm{w}))}} .
$$


We therefore have the bound

$$
\left.\frac{d Q_{\mathrm{w}_{1}}}{d Q_{\mathrm{w}_{2}}}\right|_{\mathcal{F}_{n}}(\mathcal{Z}) \leq \bar{C}^{H\left(n, S\left(\mathrm{w}_{1}\right) \cup S\left(\mathrm{w}_{2}\right), \mathcal{Z}\right)} .
$$

Notice now that if one has $\sup _{x \in S} x \cdot \ell=D<\infty$ and $n^{-1} x_{n} \rightarrow \xi$, then $\operatorname{dist}\left(x_{n}, S\right) \geq\left(x_{n} \cdot \ell-D\right) \sim n \xi \cdot \ell$ and $\sup _{n} H(n, S, \mathcal{Z})<\infty$, fulfilling the third requirement of Theorem 2.1 .

LEMMA 5.4 Condition (2.9) is satisfied.

PRoOF: Fix an $\ell \in \mathbb{S}^{d-1}$, a w $\mathrm{w}_{2} \in \mathrm{W}^{\ell}$, and a finite connected $\Lambda \subset\left\{x \in \mathbb{Z}^{d}\right.$ : $x \cdot \ell \geq 0\}$ containing 0 . Recalling (4.3), one has

$$
q\left(T_{z_{-1}} \cdots T_{z_{-m+1}} \mathrm{w}, z\right)=\frac{\mathbb{E}^{\mathbb{P}^{\mathrm{w}}}\left(\pi_{0 z_{-m+1}} \cdots \pi_{z_{-m+1}+\cdots+z_{0}, z_{-m+1}+\cdots+z_{0}+z}\right)}{\mathbb{E}^{\mathbb{P w}}\left(\pi_{0 z_{-m+1}} \cdots \pi_{z_{-m+1}+\cdots+z_{-1}, z_{-m+1}+\cdots+z_{0}}\right)} .
$$

Considering that, for any $m \geq 1$ and $\mathrm{w}_{1}=\left(z_{-m+1}, \ldots, z_{0}\right) \in \mathrm{W}_{m}^{\Lambda}$, both functions appearing inside the expectations in the above ratio are $\mathfrak{S}_{\Lambda+\{0, z\}}$-measurable, one needs only to show that

$$
\lim _{A \rightarrow \infty} \sup _{\mathrm{w}_{3} \in \cup \mathrm{W}_{m}^{\ell,-}} \sup _{\omega_{\Lambda}}\left|\frac{d \mathbb{P}_{\Lambda}^{T_{\mathrm{w}_{2}^{(A)}}}}{d \mathbb{P}_{\Lambda}^{\mathrm{w}_{2}}}\left(\omega_{\Lambda}\right)-1\right|=0 .
$$

Notice that for $V \subset \mathbb{Z}^{d}$ containing $\Lambda$, one has

$$
\sup _{\mathrm{w}_{3} \in \cup \mathrm{W}_{m}^{\ell,-}} \sup _{\omega_{\Lambda}, \omega_{V^{c}}}\left|\frac{d \mathbb{P}_{V, \Lambda}^{T_{\mathrm{w}}^{(A)} \mathrm{w}_{3}, \omega_{V^{c}}}}{d \mathbb{P}_{V, \Lambda}^{\mathrm{w}_{2}, \omega_{V^{c}}}}\left(\omega_{\Lambda}\right)-1\right|=0
$$

as soon as $A>\operatorname{dist}\left(0, V^{c}\right)$.

On the other hand, it is not hard to show that for a Gibbs field $\mathbb{Q}$ satisfying (4.1), one has

$$
\sup _{\omega_{V^{c}, \omega_{\Lambda}}}\left|\frac{d \mathbb{Q}_{\Lambda}}{d \mathbb{Q}_{V, \Lambda}^{\omega_{V^{c}}}}-1\right| \leq C_{d} G e^{-0.5 g \operatorname{dist}\left(\Lambda, V^{c}\right)},
$$

with the same inequality for $d \mathbb{Q}_{V, \Lambda}^{\omega_{V c}} / d \mathbb{Q}_{\Lambda}$; see, for example, [6, lemma 9]. But, since for each fixed $\Lambda \subset V$ and $\omega_{V^{c}}, \mathbb{P}_{V, \Lambda}^{T_{\mathrm{w}_{2}}^{(A) \mathrm{w}_{3}, \omega_{V^{c}}}}$ converges weakly to $\mathbb{P}_{V, \Lambda}^{\mathrm{w}_{2}, \omega_{V^{c}}}$ as $A$ grows to $\infty$, one can consider that, for $A$ large enough, $G\left(T_{\left.\mathrm{w}_{2}^{(A)} \mathrm{w}_{3}\right)}\right)=2 G\left(\mathrm{w}_{2}\right)$

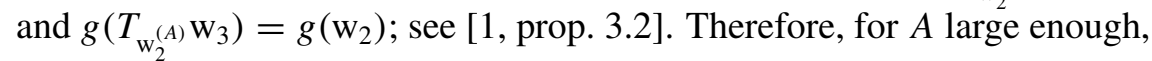

$$
\begin{aligned}
& \frac{d \mathbb{P}_{\Lambda}^{{ }_{\mathrm{w}}(A) \mathrm{w}_{3}}}{d \mathbb{P}_{\Lambda}^{\mathrm{W}_{2}}}\left(\omega_{\Lambda}\right)=\frac{d \mathbb{P}_{\Lambda}^{\mathrm{w}_{2}^{(A)} \mathrm{w}_{3}}}{T^{\mathrm{w}_{2}(A) \mathrm{w}_{3}, \omega_{V^{c}}}}\left(\omega_{\Lambda}\right) \frac{d \mathbb{P}_{V, \Lambda}^{\mathrm{W}_{2}, \omega_{V^{c}}}}{d \mathbb{P}_{\Lambda}^{\mathrm{W}_{2}}}\left(\omega_{\Lambda}\right) \leq \\
& \quad\left(1+2 \mathbb{P}_{V, \Lambda} G\left(\mathrm{w}_{2}\right) e^{-0.5 g\left(\mathrm{w}_{2}\right) \operatorname{dist}\left(\Lambda, V^{c}\right)}\right)\left(1+C_{d} G\left(\mathrm{w}_{2}\right) e^{-0.5 g\left(\mathrm{w}_{2}\right) \operatorname{dist}\left(\Lambda, V^{c}\right)}\right)
\end{aligned}
$$


with a similar inequality for the lower bound. Thus,

$$
\lim _{A \rightarrow \infty} \sup _{\mathrm{w}_{3} \in \bigcup \mathrm{w}_{m}^{\ell-}} \sup _{\omega_{\Lambda}}\left|\frac{d \mathbb{P}_{\Lambda}^{T_{2}^{(A)} \mathrm{w}_{3}}}{d \mathbb{P}_{\Lambda}^{\mathrm{W}_{2}}}\left(\omega_{\Lambda}\right)-1\right| \leq \bar{C}_{d} G\left(\mathrm{w}_{2}\right) e^{-0.5 g\left(\mathrm{w}_{2}\right) \operatorname{dist}\left(\Lambda, V^{c}\right)} .
$$

To conclude the proof, one increases $V$ to $\mathbb{Z}^{d}$.

Lemma 5.5 Condition (2.10) holds.

PROOF: Let $C_{L}$ be a sequence of finite connected subsets, all containing 0 and increasing to $\mathbb{Z}^{d}$. Fix an environment $\omega$, and consider the quenched walk $P_{0}^{\omega}$ on the countable space $\mathbb{Z}^{d}$. By standard arguments, as in [8, sec. 7], for example, we know that

$$
k=-\lim _{n \rightarrow \infty} n^{-1} \log \pi_{x x}^{(n)}(\omega)
$$

exists, is independent of $x$ and $\omega$, and is equal to $-\sup _{\omega, F} \log \rho\left(\left.\pi(\omega)\right|_{F}\right)$, where the $F$ 's are finite connected sets in $\mathbb{Z}^{d}$. Here $\left.\pi(\omega)\right|_{F}$ is the stochastic matrix that is obtained by restricting $\pi(\omega)$ to $F$, and $\rho$ is its spectral radius.

Let $F$ be such that $-\sup _{\omega} \log \rho\left(\left.\pi(\omega)\right|_{F}\right)$ is close to its minimum $k$. By ergodicity, there will be such a favorable spot $F, \mathbb{P}$-a.s. Then the rate of spending a long time in $F$ is no worse than $-\sup _{\omega} \log \rho\left(\left.\pi(\omega)\right|_{F}\right)$, which is close to $k$; see, for example, the discussion in [8, sec. 7]. This proves that $\inf _{L} \sigma_{C_{L}, \phi} \leq k$.

If one starts with a nonempty history $\mathrm{w}$ of finite length, then one can use ellipticity to clear the history. Namely, one has

$$
\left|\log \frac{d Q_{\mathrm{w}}}{d Q_{\phi}}\right| \leq|S(\mathrm{w})| \log \kappa^{-1} .
$$

One then has $\inf _{L} \sigma_{C_{L} \text {,w }} \leq k$ for all such w.

To finish the proof, for a random walk in a random environment, we notice that $k \leq H(0)$. For this, see [8, lemma 7.2].

This completes the proof of Theorem 5.1.

Acknowledgment. The author thanks Prof. S. R. S. Varadhan for suggesting this problem and for many valuable discussions.

\section{Bibliography}

[1] Dobrushin, R. L.; Shlosman, S. B. Completely analytical Gibbs fields. Statistical physics and dynamical systems (Köszeg, 1984), 371-403. Progress in Physics, 10. Birkhäuser, Boston, 1985.

[2] Dobrushin, R. L.; Shlosman, S. B. Constructive criterion for the uniqueness of a Gibbs field. Statistical physics and dynamical systems (Köszeg, 1984), 347-370. Progress in Physics, 10. Birkhäuser, Boston, 1985.

[3] Donsker, M. D.; Varadhan, S. R. S. Asymptotic evaluation of certain Markov process expectations for large time. I. Comm. Pure Appl. Math. 28 (1975), 1-47.

[4] Dugundji, J. Topology. Reprinting of the 1966 original. Allyn and Bacon Series in Advanced Mathematics. Allyn and Bacon, Boston-London-Sydney, 1978. 
[5] Georgii, H. O. Gibbs measures and phase transitions. De Gruyter, Berlin, 1988.

[6] Rassoul-Agha, F. The point of view of the particle on the law of large numbers for random walks in a mixing random environment. Ann. Probab. 31 (2003), 1441-1463.

[7] Varadhan, S. R. S. Large deviations and applications. CBMS-NSF Regional Conference Series in Applied Mathematics, 46. Society for Industrial and Applied Mathematics (SIAM), Philadelphia, 1984.

[8] Varadhan, S. R. S. Large deviations for random walks in a random environment. Comm. Pure Appl. Math. 56 (2003), 1222-1245.

F. RASSOUL-AGHA

Ohio State University

Mathematics Department

231 West $18^{\text {th }}$ Avenue

Columbus, OH, 43210-117

E-mail: firas@math.ohio-state.edu

Homepage: www.math.ohio-state.edu/ firas

Received May 2003.

Revised February 2004. 